\title{
Performance Analysis for Lossy-Forward Relaying over Nakagami-m Fading Channels
}

\author{
Shen Qian, Student Member, IEEE, Xiaobo Zhou, Member, IEEE, Xin He, Member, IEEE, Jiguang He, Student \\ Member, IEEE, Markku Juntti, Senior Member, IEEE, and Tad Matsumoto, Fellow, IEEE
}

\begin{abstract}
We investigate the performance of three-node lossyforward (LF) relaying over independent block Nakagami- $m$ fading channels. Based on the theorem of source coding with side information, the exact outage probability expression for arbitrary values of the shape factor $m$ is derived under the assumptions of both the Gaussian codebook capacity and the constellation constrained capacity. The difference in outage probability between the two codebook models of capacity is found to be very minor. Furthermore, an accurate high signal-to-noise ratio approximation for the outage probability is obtained. It clearly identifies the equivalent diversity order and coding gain of the $L F$ relaying. It is shown that the $L F$ relaying is superior to conventional decode-and-forward relaying in terms of the outage probability and the $\epsilon$-outage achievable rate. Moreover, with the $L F$ relaying, the optimal location for the relay $(R)$, which minimizes the outage probability, is found to be the point having same distance to the source (S) and the destination (D) when the S-R and R-D links experience same level of fading. The accuracy of the analytical results is verified by a series of Monte Carlo simulations.
\end{abstract}

Index Terms-outage probability, relay channels, lossy forward, Nakagami- $m$ fading, equivalent diversity order, coding gain, $\epsilon$-outage achievable rate, optimal relay location

\section{INTRODUCTION}

$\mathbf{C}$ OOPERATIVE communication is a scheme which offers spatial diversity to mitigate the effect of fading in the propagation medium [1], [2]. Among the various relaying protocols, decode-and-forward (DF) relaying has drawn significant and practical attentions, and been widely studied. Several analyses about the diversity and multiplexing gains as well as the diversity-multiplexing tradeoff of the DF relaying have been conducted for half duplex and full duplex relaying [3]-[5]. Various practical implementations of the DF relaying have been performed for single-antenna [6], [7] and multiple-antenna scenarios [8], [9]. Theoretical outage

This research was supported in part by the European Unions FP7 project, ICT-619555 RESCUE (Links-on-the-fly Technology for Robust, Efficient and Smart Communication in Unpredictable Environments), in part by the Academy of Finland NETCOBRA project, and also in part by the JAIST Doctoral Research Fellow program.

Shen Qian and Tad Matsumoto are with the School of Information Science, Japan Advanced Institute of Science and Technology (JAIST), 11 Asahidai, Nomi, Ishikawa, Japan 923-1292 (e-mail: \{shen.qian, matumoto\}@jaist.ac.jp), and the Centre for Wireless Communication, University of Oulu, FI-90014 Finland.

Xiaobo Zhou is with the School of Computer Science and Technology, Tianjin University, Tianjin, China. (e-mail: xiaobo.zhou@tju.edu.cn).

Xin He is with the School of Mathematics and Computer Science, Anhui Normal University, Anhui, China. (e-mail: xin.he@ahnu.edu.cn).

Jiguang $\mathrm{He}$ and Markku Juntti are with the Centre for Wireless Communication, University of Oulu, FI-90014 Finland (e-mail:\{jhe, markku.juntti\}@oulu.fi). probability, which provides useful performance metrics for designing practical coding techniques [10], is analyzed for the DF relaying in [11]-[13].

Recently, a new cooperative communication scheme based on the DF protocol allowing source-relay (S-R) link errors is proposed in [14]. The scheme is referred to as lossy forward (LF) relaying in this paper. Unlike the conventional DF strategy, the decoded information sequence at relay (R) is interleaved, re-encoded, and transmitted to destination (D), even though error is detected during the decoding process at $\mathrm{R}$. The two information sequences, one from the source $(S)$ and the other from $\mathrm{R}$, received at $\mathrm{D}$ are correlated. The correlation knowledge can be estimated and utilized in a joint decoder at D. For example, iterative processing between two decoders (one is for $\mathrm{S}$ and the other is for $\mathrm{R}$ ) for updating log-likelihood ratio (LLR) via a LLR modification function, proposed in [15], reduces the decoding error probability. The basic idea behind the LF relaying is the relay system, as a whole, can be seen as a distributed turbo code, and hence it can achieve turbocliff-like bit-error-rate (BER) performance in additive white Gaussian noise (AWGN) channels [16].

The outage probability of the LF relaying has been evaluated in [17], where the admissible rate region is determined by the Slepian-Wolf correlated source coding theorem. It was found soon after [17] was published, that analyzing the exact rate region of the LF scheme falls into the source coding with side information in network information theory [18], [19]. Based on this finding, the exact and approximated outage probabilities were analyzed in [20]. It was shown that the LF relaying can achieve lower outage probability in Rayleigh fading channels compared to the DF relaying. The technique is further extended to wireless sensor networks (WSNs) and a simple, general, efficient power allocation scheme for arbitrary number of sensors is derived [21]. In [22], the outage probability of the LF relaying is analyzed, where the source-destination (S-D) link is assumed to be suffering from block Rayleigh fading, whereas the relay-destination (R-D) link from block Rician fading. Brulatout et al. propose a multiple access channel (MAC) protocol in [23] specifically designed to optimally operate with the LF paradigm.

However, there are several drawbacks in the previous work related to the LF system: 1) Performance analyses for the LF relaying are all based on numerical calculation. Diversity and coding gain cannot be identified separately from the outage probability expression; 2) In [17], [20], [22], Rayleigh and Rician distributions are assumed as fading channel models, since they are widely used to describe the statistical properties 
of the fading channels in wireless mobile communication systems. However, Nakagami- $m$ distribution is considered to be more practical and better matched to field measurement data than Rayleigh and Rician distributions [24]; 3) Even though [20] has derived the exact outage probability of the LF relaying based on the rate region determined by the theorem of source coding with side information, Gaussian codebook capacity (GCC) is assumed when describe the relationship between the Hamming distortion of the S-R link and the receive blockwise instantaneous signal-to-noise ratio (SNR). However, in practice, channel capacity is upper bounded by the modulation constellation size.

The main objective of this paper is to eliminate the aforementioned drawbacks and to theoretically investigate outage probability of the LF relaying, where all the links are assumed to experience block Nakagami- $m$ fading. The major contributions of this paper are as follows.

- The exact outage probability for the LF relaying over Nakagami- $m$ fading channels is derived. The outage expressions are further approached by accurate enough approximations in high SNR regime. Explicit expressions of the equivalent diversity order and coding gain for arbitrary values of the shape factor $m$ are then obtained from the approximated outage expressions. It is shown that the equivalent diversity order (outage curve decay) of the LF relaying is limited to either the S-R link or R-D link, having smaller shape factor $m$.

- The outage probabilities of the LF system using the GCC and constellation constrained capacity (CCC) are derived. The numerical results indicate that the difference between the GCC and CCC based outage probabilities of the LF relaying is negligible.

- We introduce another performance metric, $\epsilon$-outage achievable rate, which indicates the largest transmission rate such that the outage probability is not larger than a threshold value $\epsilon$ [25], [26]. We identify from the analytical results that in high SNR region, the $\epsilon$-outage achievable rate of the $\mathrm{LF}$ relaying increases rapider than that of the conventional DF relaying.

- The optimal relay location is investigated for minimizing the outage probability of the LF relaying. The relay $\mathrm{R}$ is considered to be located on a line parallel to the S-D link, as shown in Fig. 1. It is shown that the optimal relay location is always at the point where the S-R and R-D links have equal distance, as long as the values of shape factor $m$ of the S-R and R-D links are equivalent.

The rest of this paper is organized as follows: the system model used to analyze the outage probability of the LF relaying is presented in Section II. The admissible rate region and outage probability definition are described in Section III. The exact outage probabilities of the LF relaying based on GCC and CCC are also derived in Section III. Equivalent diversity order and coding gain with the LF relaying are provided in Section IV. In Section V, $\epsilon$-outage achievable rate is analyzed. The optimal relay location is investigated in Section VI. Finally, the conclusion is drawn in Section VII.

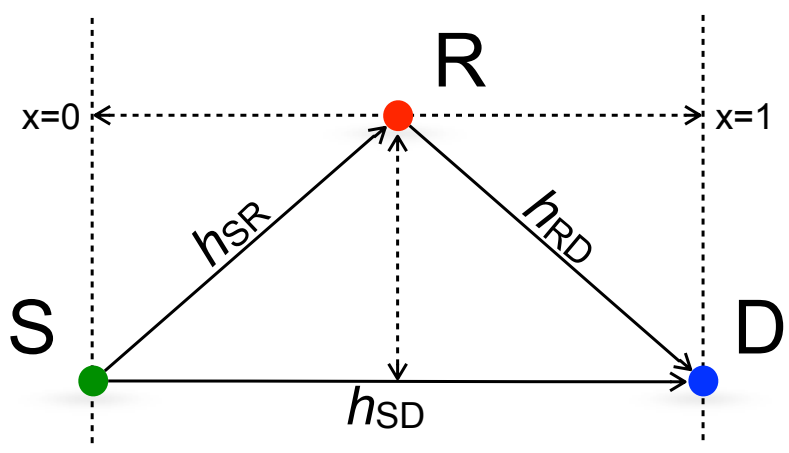

Fig. 1. The schematic of one relay aided communication system.

\section{SySTEM MOdel}

We consider a simple three-node relaying system as shown in Fig. 1. The source S communicates with the destination D with the help of a single relay $\mathrm{R}$. The location of $\mathrm{R}$ varies in a line parallel to the S-D link between $x=0$ (nearest to $\mathrm{S}$ ) and $x=1$ (nearest to $\mathrm{D}$ ). We assume time-division transmission, where the overall transmission is divided into two time slots. At the first time slot, the original uniformly distributed binary information sequence $b_{\mathrm{S}}$ is broadcast from $\mathrm{S}$. The relay $\mathrm{R}$ aims to recover the information sequence and transmits it to $\mathrm{D}$ at the second time slot, i.e., in orthogonal transmission. S does not transmit during the second time slot.

\section{A. LF Relaying}

In conventional DF relaying, $\mathrm{R}$ keeps silent if error/errors is/are detected after decoding in the information sequence sent through the S-R link. With the LF relaying, after receiving the signal from $\mathrm{S}, \mathrm{R}$ attempts to recover $b_{\mathrm{S}}$. Although the decoding result of $b_{\mathrm{S}}$ at $\mathrm{R}$, denoted by $b_{\mathrm{R}}$, may contain errors, $\mathrm{R}$ interleaves the information sequence $b_{\mathrm{R}}$, re-encodes the interleaved sequence, and forwards it to $\mathrm{D}$.

When only the information sequence is concerned before encoding at $\mathrm{S}$ and after decoding at $\mathrm{R}$, the $\mathrm{S}-\mathrm{R}$ link can be virtually modeled by a binary symmetric channel (BSC) model with a crossover probability $p_{f}$. More specifically, $p_{f}$ represents the bit flipping probability between the information sequence obtained after decoding at $\mathrm{R}$ and the original information sequence sent from S. Hence, $b_{\mathrm{R}}=b_{\mathrm{S}} \oplus e$, where $\oplus$ denotes the modulo- 2 addition and $e$ is a realization of a binary random variable $E$ with $\operatorname{Pr}(E=1)=p_{f}$ which stays the same within each block while changes block-by-block with its value determined by the instantaneous SNR.

At $\mathrm{D}$, after receiving the signals from $\mathrm{S}$ and $\mathrm{R}$, joint decoding is performed to retrieve the original information $b_{\mathrm{S}}$. Iterative decoding is utilized between two decoders for decoding the messages sent from $\mathrm{S}$ and $\mathrm{R}$. During the decoding process, the S-R link error probabilities can be estimated at D by making a comparison between the LLRs output from the two decoders. The estimated $p_{f}$ value is used as the correlation knowledge between $b_{\mathrm{S}}$ and $b_{\mathrm{R}}$ [14]. The LLRs of the systematic bits are exchanged between the two decoders via the interleaver/de-interleaver. Therefore, the system, as a whole, can be viewed as a distributed turbo code. 


\section{B. Channel Model}

The signals received at $\mathrm{D}$ and $\mathrm{R}$ during the first time slot, $y_{\mathrm{D}, 1}$ and $y_{\mathrm{R}, 1}$, respectively, and the signal received at $\mathrm{D}$ in the second time slot, $y_{\mathrm{D}, 2}$, are expressed as ${ }^{1}$

$$
\begin{aligned}
& y_{\mathrm{D}, 1}=\sqrt{G_{\mathrm{SD}}} h_{\mathrm{SD}} x_{1}+n_{\mathrm{D}, 1}, \\
& y_{\mathrm{R}, 1}=\sqrt{G_{\mathrm{SR}}} h_{\mathrm{SR}} x_{1}+n_{\mathrm{R}, 1}, \\
& y_{\mathrm{D}, 2}=\sqrt{G_{\mathrm{RD}}} h_{\mathrm{RD}} x_{2}+n_{\mathrm{D}, 2},
\end{aligned}
$$

respectively, where $G_{i j}(i \in(\mathrm{S}, \mathrm{R}), j \in(\mathrm{R}, \mathrm{D}), i \neq j)$ are the geometric gains related to the transmit distance of each link. $x_{1}$ and $x_{2}$ denote the modulated symbols corresponding to the coded and interleaved information sequence, transmitted from S and R, respectively. $h_{i j}$ denotes the complex channel gain and $n_{j, 1}$ and $n_{j, 2}$ are zero-mean AWGN with variance of $N_{0} / 2$ per dimension. It is assumed that $E\left[\left|h_{i j}\right|^{2}\right]=1$ and $h_{i j}$ stays constant over one block duration due to the block fading assumption.

The transmit power of each symbol is denoted as $E_{s}$. Therefore, the average and instantaneous SNRs are expressed as $\bar{\gamma}_{i j}=G_{i j} \frac{E_{s}}{N_{0}}$ and $\gamma_{i j}=\left|h_{i j}\right|^{2} \bar{\gamma}_{i j}(i \in(\mathrm{S}, \mathrm{R}), j \in(\mathrm{R}, \mathrm{D}), i \neq$ $j$ ), respectively. For the sake of simplicity, the variations due to shadowing and the fading frequency selectivity are not taken into account.

We assume that all the links (i.e., the S-R, S-D, and RD links) suffer from independent block Nakagami- $m$ fading, with the probability density function (pdf) of $\gamma_{i j}$ given by

$$
p\left(\gamma_{i j}\right)=\frac{m_{i j}^{m_{i j}}\left(\gamma_{i j}\right)^{m_{i j}-1}}{\left(\bar{\gamma}_{i j}\right)^{m_{i j}} \Gamma\left(m_{i j}\right)} \exp \left(-\frac{m_{i j} \gamma_{i j}}{\bar{\gamma}_{i j}}\right), m_{i j}>0.5,
$$

where $\Gamma(\cdot)$ is the Gamma function. The shape factor $m_{i j}$ represents the severity of the fading variation of the corresponding link.

In cooperative networks such as vehicle-to-vehicle (V2V) and/or vehicle-to-infrastructure (V2I) communications, the network topology and the fading distributions of the links change over time. A reasonable scenario assumption is that S-D link suffers from severe fading, the destination needs the help of a relay via S-R and R-D links, which suffer from moderate fading. Hence, we set the shape factor of the SD link $m_{\mathrm{SD}}=1$, corresponding to Rayleigh fading, while for other links (the S-R and R-D links), we set their corresponding $m$ values arbitrarily as parameters.

\section{Outage Probability Analysis}

In this section, the definition of admissible rate region and the derivation for outage probability of the LF relaying are provided. The outage probabilities are derived based on both GCC and CCC assumptions.

\section{A. Source Coding with Side Information based Admissible Rate Region for $S$ and $R$}

Since D aims to recover $b_{\mathrm{S}}$ only, the coded signal transmitted from $\mathrm{R}$ can be considered as side information of

\footnotetext{
${ }^{1}$ The symbol indexes are omitted in (1), (2), and (3) for conciseness.
}

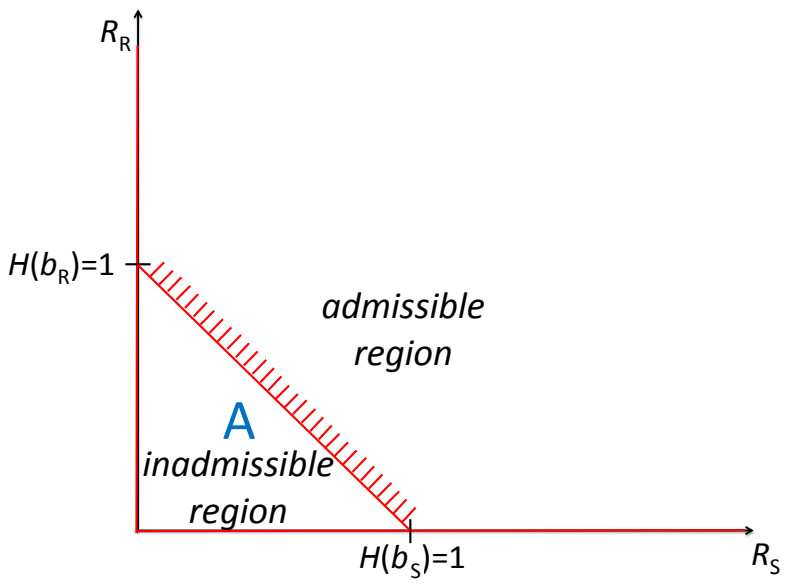

Fig. 2. Rate region for $\mathrm{S}$ and $\mathrm{R}$ when $p_{f}=0$; the red solid line with bars separates the admissible and inadmissible regions.

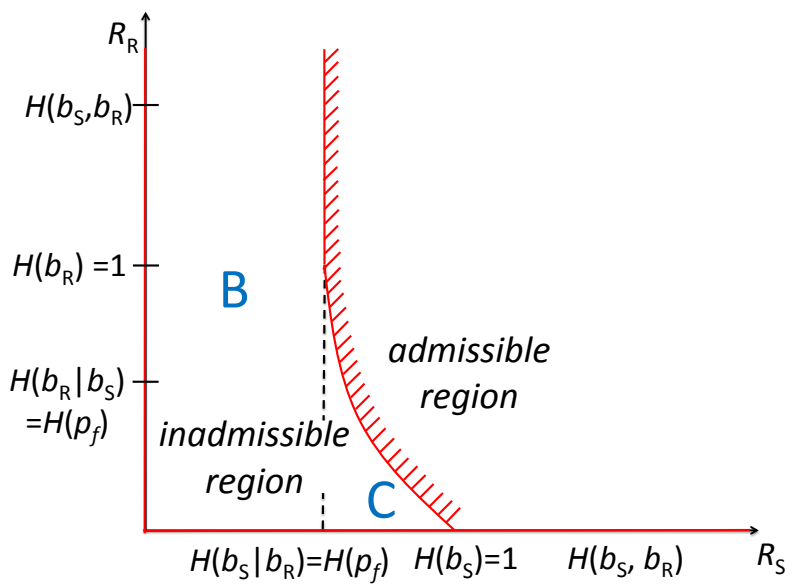

Fig. 3. Rate region for $\mathrm{S}$ and $\mathrm{R}$ when $p_{f} \neq 0$; the red solid line with bars separates the admissible and inadmissible regions.

$b_{\mathrm{S}}$. According to the theorem of source coding with side information [19, Section10.4], D can recover $b_{\mathrm{S}}$ losslessly, if the source coding rate pair of $\mathrm{S}$ and $\mathrm{R}, R_{\mathrm{S}}$ and $R_{\mathrm{R}}$, satisfies

$$
\left\{\begin{array}{l}
R_{\mathrm{S}} \geq H\left(b_{\mathrm{S}} \mid \hat{b}_{\mathrm{R}}\right), \\
R_{\mathrm{R}} \geq I\left(b_{\mathrm{R}} ; \hat{b}_{\mathrm{R}}\right),
\end{array}\right.
$$

where $H(\cdot \mid \cdot)$ and $I(\cdot ; \cdot)$ denote the conditional entropy and the mutual information between their arguments, respectively, and $\hat{b}_{\mathrm{R}}$ is the estimate of $b_{\mathrm{R}}$ obtained at D.

With the block fading assumption, we use a BSC model to represent the R-D link (helper channel), block by block, as $\hat{b}_{\mathrm{R}}=b_{\mathrm{R}} \oplus e^{\prime}$ with $\operatorname{Pr}\left(E^{\prime}=1\right)=p_{f}^{\prime}$, where $e^{\prime}$ is a realization of a binary random variable $E^{\prime}$. Since the source is assumed to be binary, uniform, and independently and identically distributed (i.i.d), (5) can be expressed as

$$
\left\{\begin{array}{l}
R_{\mathrm{S}} \quad \geq H\left(p_{f} * p_{f}^{\prime}\right) \\
R_{\mathrm{R}} \geq H\left(\hat{b}_{\mathrm{R}}\right)-H\left(\hat{b}_{\mathrm{R}} \mid b_{\mathrm{R}}\right)=1-H\left(p_{f}^{\prime}\right)
\end{array}\right.
$$

where $p_{f} * p_{f}^{\prime}=\left(1-p_{f}\right) p_{f}^{\prime}+\left(1-p_{f}^{\prime}\right) p_{f}$ and $H(\cdot)$ denotes the binary entropy function. 
$p_{f}=0$ indicates perfect decoding at $\mathrm{R}$, and hence $H\left(b_{\mathrm{S}} \mid b_{\mathrm{R}}\right)=H\left(b_{\mathrm{R}} \mid b_{\mathrm{S}}\right)=0$. In this case, the inadmissible rate region becomes the triangle area $\mathrm{A}$ as shown in Fig. 2. When $0<p_{f} \leq 0.5$, the inadmissible region, which can be divided into two areas, $\mathrm{B}$ and $\mathrm{C}$, is shown in Fig. 3. The rate region defined in (6) indicates that, even with $0 \leq R_{\mathrm{R}} \leq H\left(b_{\mathrm{R}}\right)$, $b_{\mathrm{R}}$ can be partially recovered at $\mathrm{D}$, and $b_{\mathrm{R}}$ containing errors can serve as the side information for losslessly recovering $b_{\mathrm{S}}$. In the case $R_{\mathrm{R}}>H\left(b_{\mathrm{R}}\right)$, the conditions in (6) become to $R_{\mathrm{S}} \geq H\left(p_{f}\right)$ and $R_{\mathrm{R}} \geq 1$.

\section{B. Outage Event of $L F$ relaying}

If the rate pair $\left(R_{\mathrm{S}}, R_{\mathrm{R}}\right)$ falls into the inadmissible regions in Fig. 2 or Fig. 3, the outage event occurs and D cannot guarantee the reconstruction of $b_{\mathrm{S}}$ with an arbitrarily small error probability. Since $p_{f}=0$ and $0<p_{f} \leq 0.5$ are distinctive, the outage probability of the LF relaying can be expressed as

$$
P_{\text {out }}^{\mathrm{LF}}=P_{\mathrm{A}}+P_{\mathrm{B}}+P_{\mathrm{C}}
$$

where $P_{\mathrm{A}}, P_{\mathrm{B}}$, and $P_{\mathrm{C}}$ denote the probabilities that $\left(R_{\mathrm{S}}, R_{\mathrm{R}}\right)$ falls into the inadmissible areas $\mathrm{A}, \mathrm{B}$, and $\mathrm{C}$, respectively. Taking into account the impact of $p_{f}$ and $p_{f}^{\prime}, P_{\mathrm{A}}, P_{\mathrm{B}}$, and $P_{\mathrm{C}}$ can further be expressed as

$$
\begin{aligned}
& P_{\mathrm{A}}=\operatorname{Pr}\left[p_{f}=0,0 \leq R_{\mathrm{S}}<1,0 \leq R_{\mathrm{R}}<H\left(p_{f} * p_{f}^{\prime}\right)\right], \\
& P_{\mathrm{B}}=\operatorname{Pr}\left[0<p_{f} \leq 0.5,0 \leq R_{\mathrm{S}}<H\left(p_{f}\right), R_{\mathrm{R}} \geq 0\right] \\
& P_{\mathrm{C}}=\operatorname{Pr}\left[0<p_{f} \leq 0.5, H\left(p_{f}\right) \leq R_{\mathrm{S}}<1,\right. \\
& \left.\quad 0 \leq R_{\mathrm{R}}<H\left(p_{f} * p_{f}^{\prime}\right)\right] .
\end{aligned}
$$

1) Outage Derivation with Gaussian Codebook Capacity $(G C C)$ : For calculating the outage probability, first we establish the relationship between $\gamma_{\mathrm{SD}}$ and $R_{\mathrm{S}}$, and that between $\gamma_{\mathrm{RD}}$ and $R_{\mathrm{R}}$. According to the Shannon's lossless source channel separation theorem [27], if

$$
R_{i} \cdot R_{c, i \mathrm{D}} \leq C_{i \mathrm{D}},(i \in \mathrm{S}, \mathrm{R})
$$

is satisfied, the error probability can be arbitrarily small at the destination. $C_{i \mathrm{D}}$ and $R_{c, i \mathrm{D}}$, respectively, denote the channel capacity of the $i$-D link and the normalized spectrum efficiency of the corresponding transmission chain. The spectrum efficiency includes the channel coding rate and the modulation constellation size. With the assumption that Gaussian codebook is used, the channel capacity $C_{i \mathrm{D}}$ of each link can be expressed as

$$
C_{i \mathrm{D}}=\frac{E^{n}}{2} \log _{2}\left(1+\frac{2 \gamma_{i \mathrm{D}}}{E^{n}}\right),(i \in \mathrm{S}, \mathrm{R}),
$$

where $E^{n}$ denotes the signaling dimensionality. Hence, the relationship between the instantaneous channel SNR $\gamma_{i \mathrm{D}}$ and its corresponding source coding rate $R_{i}$ is given by

$$
R_{i} \leq \Theta\left(\gamma_{i \mathrm{D}}\right)=\frac{E^{n}}{2 R_{c, i \mathrm{D}}} \log _{2}\left(1+\frac{2 \gamma_{i \mathrm{D}}}{E^{n}}\right),(i \in \mathrm{S}, \mathrm{R})
$$

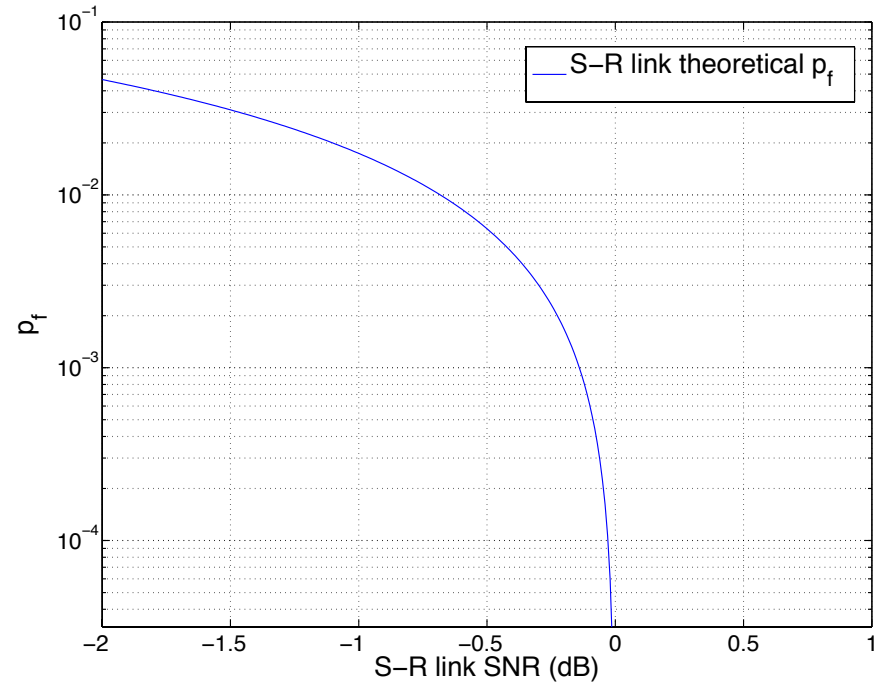

Fig. 4. S-R link Hamming distortion $p_{f}$ versus SNR, where $E_{n}=2$ and $R_{c, \mathrm{SR}}=1$.

with its inverse inequality

$$
\gamma_{i \mathrm{D}} \geq \Theta^{-1}\left(R_{i}\right)=\frac{E^{n}}{2}\left(2^{\frac{2 R_{i} R_{c, i \mathrm{D}}}{E^{n}}}-1\right)
$$

Then, we establish the relationship between $\gamma_{\mathrm{SR}}$ and $p_{f}$. Since $p_{f}$ only depends on the quality of the S-R link, according to Shannon's lossy source channel separation theorem [28], we have

$$
R(\mathcal{D}) \cdot R_{c, \mathrm{SR}} \leq C_{\mathrm{SR}}
$$

where $R(\mathcal{D})$ denotes the source rate-distortion function with the distortion measure $\mathcal{D}$. For the Hamming distortion measure, $R(\mathcal{D})=1-H(\mathcal{D})$. Following [29], we set $p_{f}$ to the minimum value of $\mathcal{D}$ yielding the specified rate $R(\mathcal{D})$ in $(15)$ for a specific instantaneous S-R link SNR $\gamma_{\mathrm{SR}}$.

Since we assume that Gaussian codebook is used for the S-R link transmission, the relationship between the required instantaneous channel SNR $\gamma_{\mathrm{SR}}$ and its corresponding source rate $R(\mathcal{D})$ with distortion $\mathcal{D}$ is given by

$$
\gamma_{\mathrm{SR}} \geq \frac{E^{n}}{2}\left(2^{\frac{2 R(\mathcal{D}) R_{c, \mathrm{SR}}}{E^{n}}}-1\right)
$$

Then, we can obtain the relationship between $p_{f}$ and $\gamma_{\mathrm{SR}}$ as

$$
p_{f}=H^{-1}\left(1-\frac{\frac{E^{n}}{2} \log _{2}\left(1+\frac{2 \gamma_{\mathrm{SR}}}{E^{n}}\right)}{R_{c, \mathrm{SR}}}\right) \text {, }
$$

with $H^{-1}(\cdot)$ denoting the inverse function of $H(\cdot)$. The relationship between the S-R link SNR and $p_{f}$ is shown in Fig. 4.

With the assumption that each link suffers from statistically independent block Nakagami- $m$ fading, each term of the outage probability expression with the LF relaying in (7) can 
be expressed as

$$
\begin{aligned}
& P_{\mathrm{A}}=\operatorname{Pr}\left[\gamma_{\mathrm{SR}}>\Theta^{-1}(1), \Theta^{-1}(0)<\gamma_{\mathrm{SD}} \leq \Theta^{-1}(1),\right. \\
& \left.\Theta^{-1}(0) \leq \gamma_{\mathrm{RD}}<\Theta^{-1}\left(1-\Theta\left(\gamma_{\mathrm{SD}}\right)\right)\right] \\
& =\int_{\gamma_{\mathrm{SR}}=\Theta^{-1}(1)}^{\Theta^{-1}(\infty)} \int_{\gamma_{\mathrm{SD}}=\Theta^{-1}(0)}^{\Theta^{-1}(1)} \int_{\gamma_{\mathrm{RD}}=\Theta^{-1}(0)}^{\Theta^{-1}\left(1-\Theta\left(\gamma_{\mathrm{SD}}\right)\right)} \\
& p\left(\gamma_{\mathrm{SR}}\right) p\left(\gamma_{\mathrm{SD}}\right) p\left(\gamma_{\mathrm{RD}}\right) d \gamma_{\mathrm{SR}} d \gamma_{\mathrm{SD}} d \gamma_{\mathrm{RD}} \\
& =\frac{1}{\bar{\gamma}_{\mathrm{SD}}}\left(1-\left[\frac{\gamma\left(m_{\mathrm{SR}}, m_{\mathrm{SR}} \frac{1}{\bar{\gamma}_{\mathrm{SR}}}\right)}{\Gamma\left(m_{\mathrm{SR}}\right)}\right]\right) \\
& \cdot \int_{\gamma_{\mathrm{SD}}=\Theta^{-1}(0)}^{\Theta^{-1}(1)} \exp \left(-\frac{\gamma_{\mathrm{SD}}}{\bar{\gamma}_{\mathrm{SD}}}\right) \\
& \cdot\left[\frac{\gamma\left(m_{\mathrm{RD}}, m_{\mathrm{RD}} \frac{\Theta^{-1}\left(1-\Theta\left(\gamma_{\mathrm{SD}}\right)\right)}{\bar{\gamma}_{\mathrm{RD}}}\right)}{\Gamma\left(m_{\mathrm{RD}}\right)}\right] d \gamma_{\mathrm{SD}}, \\
& P_{\mathrm{B}}=\operatorname{Pr}\left[\Theta^{-1}(0) \leq \gamma_{\mathrm{SR}} \leq \Theta^{-1}(1), \Theta^{-1}(0)<\gamma_{\mathrm{SD}}\right. \\
& \left.\leq \Theta^{-1}\left(1-\Theta\left(\gamma_{\mathrm{SR}}\right)\right), \gamma_{\mathrm{RD}} \geq \Theta^{-1}(0)\right] \\
& =\int_{\gamma_{\mathrm{SR}}=\Theta^{-1}(0)}^{\Theta^{-1}(1)} \int_{\gamma_{\mathrm{SD}}=\Theta^{-1}(0)}^{\Theta^{-1}\left(1-\Theta\left(\gamma_{\mathrm{SR}}\right)\right)} \int_{\gamma_{\mathrm{RD}}=\Theta^{-1}(0)}^{\Theta^{-1}(\infty)} \\
& p\left(\gamma_{\mathrm{SR}}\right) p\left(\gamma_{\mathrm{SD}}\right) p\left(\gamma_{\mathrm{RD}}\right) d \gamma_{\mathrm{SR}} d \gamma_{\mathrm{SD}} d \gamma_{\mathrm{RD}} \\
& =\int_{\gamma_{\mathrm{SR}}=\Theta^{-1}(0)}^{\Theta^{-1}(1)} \frac{m_{\mathrm{SR}}^{m_{\mathrm{SR}}}\left(\gamma_{\mathrm{SR}}\right)^{m_{\mathrm{SR}}-1}}{\left(\bar{\gamma}_{\mathrm{SR}}\right)^{m_{\mathrm{SR}}} \Gamma\left(m_{\mathrm{SR}}\right)} \exp \left(-\frac{m_{\mathrm{SR}} \gamma_{\mathrm{SR}}}{\bar{\gamma}_{\mathrm{SR}}}\right) \\
& \cdot\left[1-\exp \left(-\frac{\Theta^{-1}\left(1-\Theta\left(\gamma_{\mathrm{SR}}\right)\right)}{\bar{\gamma}_{\mathrm{SD}}}\right)\right] d \gamma_{\mathrm{SR}}
\end{aligned}
$$

and

$$
\begin{aligned}
P_{\mathrm{C}}= & \operatorname{Pr}\left\{\Theta^{-1}(0) \leq \gamma_{\mathrm{SR}} \leq \Theta^{-1}(1), \Theta^{-1}\left[1-\Theta\left(\gamma_{\mathrm{SR}}\right)\right]\right. \\
& \left.<\gamma_{\mathrm{SD}} \leq \Theta^{-1}(1), \Theta^{-1}(0) \leq \gamma_{\mathrm{RD}} \geq \Theta^{-1}\left[\xi\left(\gamma_{\mathrm{SD}}, \gamma_{\mathrm{SR}}\right)\right]\right\} \\
= & \int_{\gamma_{\mathrm{SR}}=\Theta^{-1}(0)}^{\Theta^{-1}(1)} \int_{\gamma_{\mathrm{SD}}=\Theta^{-1}\left(1-\Theta\left(\gamma_{\mathrm{SR}}\right)\right)}^{\Theta^{-1}(1)} \int_{\gamma_{\mathrm{RD}}=\Theta^{-1}(0)} \\
& \left.p\left(\gamma_{\mathrm{SR}}\right) p\left(\gamma_{\mathrm{SD}}\right) p\left(\gamma_{\mathrm{RD}}\right) d \gamma_{\mathrm{SR}} d \gamma_{\mathrm{SD}} d \gamma_{\mathrm{RD}}\right] \\
= & \frac{1}{\bar{\gamma}_{\mathrm{SD}}} \int_{\gamma_{\mathrm{SR}}=\Theta^{-1}(0)}^{\Theta^{-1}(1)} \int_{\gamma_{\mathrm{SD}}=\Theta^{-1}\left(1-\Theta\left(\gamma_{\mathrm{SR}}\right)\right)}^{\Theta^{-1}(1)} \\
& \exp \left(\frac{-\gamma_{\mathrm{SD}}}{\bar{\gamma}_{\mathrm{SD}}}\right) \frac{m_{\mathrm{SR}}^{m_{\mathrm{SR}}}\left(\gamma_{\mathrm{SR}}\right)^{m_{\mathrm{SR}}-1}}{\left(\bar{\gamma}_{\mathrm{SR}}\right)^{m_{\mathrm{SR}}} \Gamma\left(m_{\mathrm{SR}}\right)} \exp \left(-\frac{m_{\mathrm{SR}} \gamma_{\mathrm{SR}}}{\bar{\gamma}_{\mathrm{SR}}}\right) \\
& \cdot\left[\frac{\gamma\left(m_{\mathrm{RD}}, m_{\mathrm{RD}} \frac{\xi\left(\gamma_{\mathrm{SD}}, \gamma_{\mathrm{SR}}\right)}{\bar{\gamma}_{\mathrm{RD}}}\right)}{\Gamma\left(m_{\mathrm{RD}}\right)}\right] d \gamma_{\mathrm{SD}} d \gamma_{\mathrm{SR}},
\end{aligned}
$$

where $\xi\left(\gamma_{\mathrm{SD}}, \gamma_{\mathrm{SR}}\right)=H\left\{H^{-1}\left[1-\Theta\left(\gamma_{\mathrm{SD}}\right)\right] * H^{-1}[1-\right.$ $\left.\left.\Theta\left(\gamma_{\mathrm{SR}}\right)\right]\right\}$ and $\gamma(\cdot, \cdot)$ is the lower incomplete gamma function.

Fig. 5 presents the outage probabilities of the LF relaying, denoted as $P_{\text {out }}^{\mathrm{LF}}$. As a reference, the outage probability of a conventional DF system, denoted as $P_{\mathrm{out}}^{\mathrm{DF}}$, is also plotted. It is found that LF achieves lower outage probability than DF. This is because LF always forwards information sequences from the relay to destination, and it converts the system into a distributed turbo code. Note that the theoretical outage probabilities exactly match their corresponding simulation results obtained by Monte Carlo method.

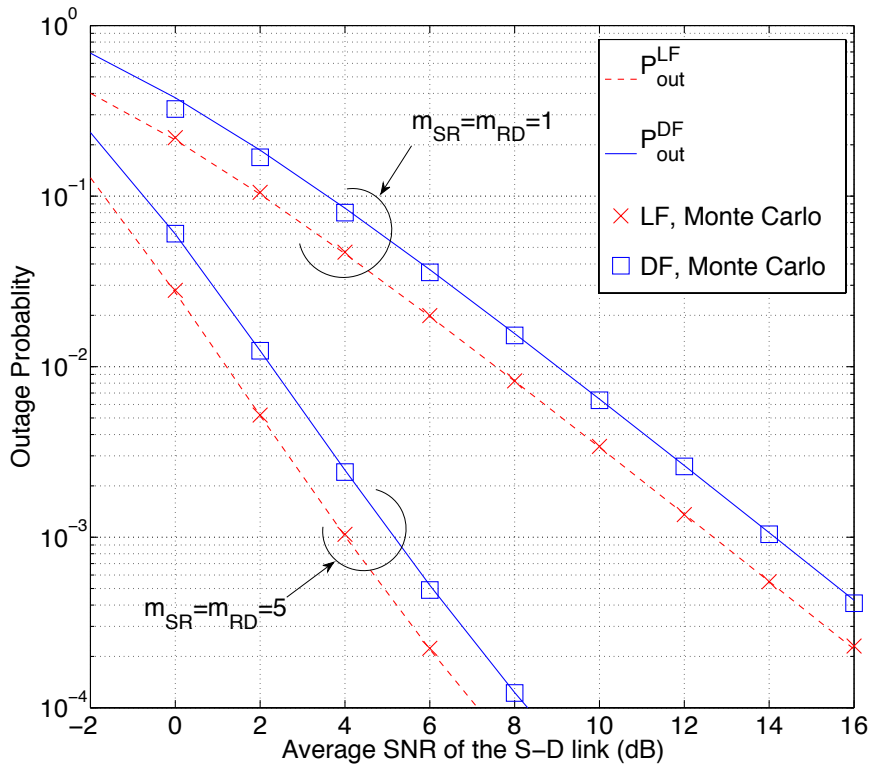

Fig. 5. Outage probability comparison between $\mathrm{LF}$ and $\mathrm{DF}$, where $m_{\mathrm{SD}}=1$ and $G_{\mathrm{SD}}=G_{\mathrm{RD}}=G_{\mathrm{SR}}$.

2) Outage Derivation with Constellation Constrained Capacity (CCC): According to Shannon's source channel separation theorem, if $R_{i} \cdot R_{c, i \mathrm{D}} \leq C_{c c}\left(\gamma_{i \mathrm{D}}\right),(i \in \mathrm{S}, \mathrm{R})$ is satisfied, the error probability can be made arbitrarily small at the destination, where $C_{c c}\left(\gamma_{i \mathrm{D}}\right)$ is the channel $\mathrm{CCC}$ with the instantaneous SNR $\gamma_{i \mathrm{D}}$ of the $i$-D link. Since the CCC has no closed-form expression, numerical evaluation yields a relationship between $\gamma_{i \mathrm{D}}$ and $R_{i}$ as $\gamma_{i \mathrm{D}} \geq C_{c c}^{-1}\left(R_{i} \cdot R_{c, i \mathrm{D}}\right)$, where $C_{c c}^{-1}$ represents the inverse function of channel CCC.

Similarly, the relationship between $\gamma_{\mathrm{SR}}$ and $p_{f}$ is given by $R(\mathcal{D}) \cdot R_{c, \mathrm{SR}} \leq C_{c c}\left(\gamma_{\mathrm{SR}}\right)$ and $\gamma_{\mathrm{SR}} \geq C_{c c}^{-1}\left(R(\mathcal{D}) \cdot R_{c, \mathrm{SR}}\right)$, where the minimum distortion $\mathcal{D}$ is equivalent to $p_{f}$ for a given instantaneous S-R link SNR $\gamma_{\mathrm{SR}}$. Then, by using the method presented in the previous subsection, the outage probability of the LF relaying with CCC can be calculated through a lookup table at each SNR value.

Fig. 6 compares the outage probabilities of the LF relaying derived from GCC and CCC, $P_{\text {out }}^{\mathrm{GCC}}$ and $P_{\text {out }}^{\mathrm{CCC}}$, respectively. ${ }^{2}$ The quaternary CCC is calculated via Monte Carlo simulations to create a lookup table. The Monte Carlo simulation results of GCC based LF relaying are also presented. We can observe from Fig. 6 that the theoretical outage probabilities with the LF relaying, calculated by using numerical integral in (18), (19), and (20), well match their corresponding Monte Carlo simulation results. It is also found that, the difference between $P_{\text {out }}^{\mathrm{GCC}}$ and $P_{\mathrm{out}}^{\mathrm{CCC}}$ is negligible. This is because in low SNR regime the difference between GCC and CCC is very small. On the contrary, in high SNR region, even though the difference between GCC and CCC becomes large, $p_{f}$ approaches zero very quickly, resulting in lossless transmission over the S-R link. Therefore, the difference between GCC and CCC based outage probabilities is negligibly small.

\footnotetext{
${ }^{2}$ In the numerical calculation, $E^{n}=2$ and, without loss of generality, $R_{c, i j}=1(i \in(\mathrm{S}, \mathrm{R}), j \in(\mathrm{R}, \mathrm{D}), i \neq j)$.
} 


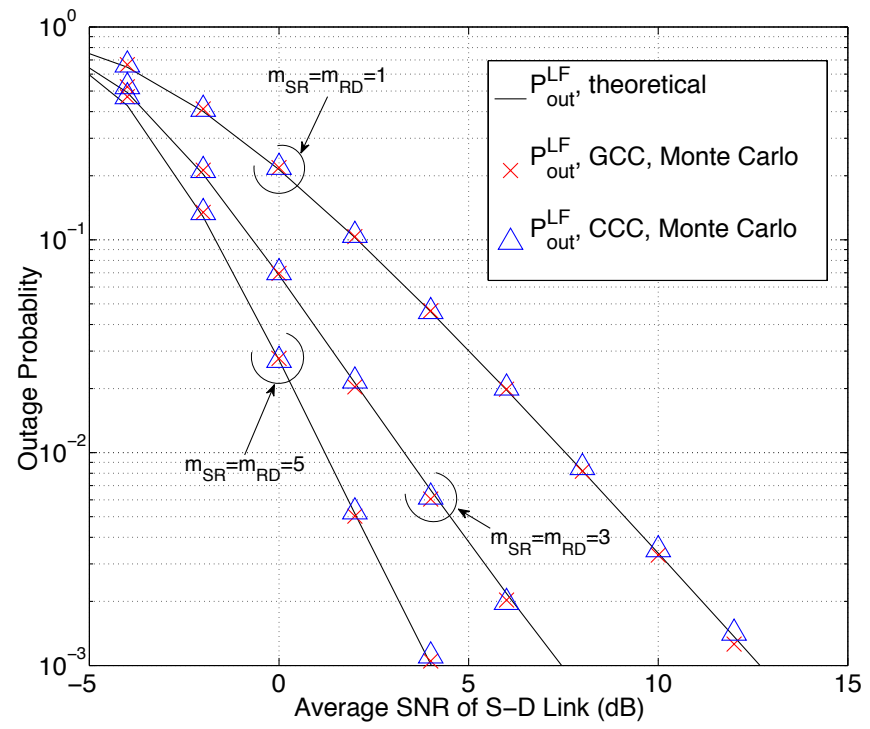

Fig. 6. Comparison of the GCC and CCC based outage probability of the $\mathrm{LF}$ relaying, where $m_{\mathrm{SR}}=m_{\mathrm{RD}}, m_{\mathrm{SD}}=1$, and $G_{\mathrm{SD}}=G_{\mathrm{RD}}=G_{\mathrm{SR}}$.

\section{Equivalent Diversity ORder And Coding Gain}

The equivalent diversity order and coding gain can be obtained by approximating (18), (19), and (20) at high SNR region. Invoking the series representation of incomplete gamma function $\gamma(a, x)=\sum_{n=0}^{\infty} \frac{(-1)^{n} x^{a+n}}{n !(a+n)}$ [30, equation 8.354.1], together with the approximation $p\left(\gamma_{i j}\right) \approx \frac{m_{i j}^{m_{i j}}\left(\gamma_{i j}\right)^{m_{i j}-1}}{\left(\bar{\gamma}_{i j}\right)^{m_{i j}} \Gamma\left(m_{i j}\right)}$ [31], after several steps of mathematical manipulations, each term of the outage probability expression with the LF relaying over Nakagami- $m$ fading channel can be approximated as

$$
\begin{aligned}
& P_{\mathrm{A}} \approx A \cdot A^{\prime} \cdot \bar{\gamma}_{\mathrm{SD}}^{\left(-m_{\mathrm{SD}}\right)} \bar{\gamma}_{\mathrm{RD}}^{\left(-m_{\mathrm{RD}}\right)} \\
& P_{\mathrm{B}} \approx B \cdot \bar{\gamma}_{\mathrm{SD}}^{\left(-m_{\mathrm{SD}}\right)} \bar{\gamma}_{\mathrm{SR}}^{\left(-m_{\mathrm{SR}}\right)}, \\
& P_{\mathrm{C}} \approx C \cdot \bar{\gamma}_{\mathrm{SD}}^{\left(-m_{\mathrm{SD}}\right)} \bar{\gamma}_{\mathrm{RD}}^{\left(-m_{\mathrm{RD}}\right)} \bar{\gamma}_{\mathrm{SR}}^{\left(-m_{\mathrm{SR}}\right)},
\end{aligned}
$$

where

$$
\begin{aligned}
A= & \frac{m_{\mathrm{SD}}^{m_{\mathrm{SD}}} m_{\mathrm{RD}}^{m_{\mathrm{RD}}-1}}{\Gamma\left(m_{\mathrm{SD}}\right) \Gamma\left(m_{\mathrm{RD}}\right)} \int_{\gamma_{\mathrm{SD}}=\Theta^{-1}(0)}^{\Theta^{-1}(1)} \frac{\left(\frac{2}{1+\gamma_{\mathrm{SD}}}-1\right)^{m_{\mathrm{RD}}}}{\gamma_{\mathrm{SD}}^{1-m_{\mathrm{SD}}}} d \gamma_{\mathrm{SD}} \\
B= & \frac{m_{\mathrm{SR}}^{m_{\mathrm{SR}}} m_{\mathrm{SD}}^{m_{\mathrm{SD}}-1}}{\Gamma\left(m_{\mathrm{SD}}\right) \Gamma\left(m_{\mathrm{SR}}\right)} \int_{\gamma_{\mathrm{SR}}=\Theta^{-1}(0)}^{\Theta^{-1}(1)} \frac{\left(\frac{2}{1+\gamma_{\mathrm{SR}}}-1\right)^{m_{\mathrm{SD}}}}{\gamma_{\mathrm{SR}}^{m_{\mathrm{SR}}-1}} d \gamma_{\mathrm{SR}} \\
C= & \int_{\gamma_{\mathrm{SR}}=\Theta^{-1}(0)}^{\Theta^{-1}(1)} \int_{\gamma_{\mathrm{SD}}=\Theta^{-1}\left(1-\Theta\left(\gamma_{\mathrm{SR}}\right)\right)}^{\Theta_{\mathrm{SD}}^{-1}(1)} \gamma_{\mathrm{SR}}^{m_{\mathrm{SR}}-1} \\
& \frac{m_{\mathrm{SR}}^{m_{\mathrm{SR}}} m_{\mathrm{SD}}^{m_{\mathrm{SD}}} m_{\mathrm{RD}}^{m_{\mathrm{RD}}-1}}{\Gamma\left(m_{\mathrm{SD}}\right) \Gamma\left(m_{\mathrm{RD}}\right) \Gamma\left(m_{\mathrm{SR}}\right)} \xi^{m_{\mathrm{SD}}}\left(\gamma_{\mathrm{SD}}, \gamma_{\mathrm{SR}}\right) d \gamma_{\mathrm{SR}} d \gamma_{\mathrm{SD}}
\end{aligned}
$$

$A^{\prime}=1-\frac{\gamma\left(m_{\mathrm{SR}}, m_{\mathrm{SR}} \frac{1}{\bar{\gamma}_{\mathrm{SR}}}\right)}{\Gamma\left(m_{\mathrm{SR}}\right)}$ indicates the probability of $p_{f}=0$ (i.e., decoding error can be made arbitrarily small at R).

Fig. 7 shows that at high SNR region the approximated outage curves obtained from (21), (22), and (23) well match the numerically calculated curves obtained from (18), (19), and

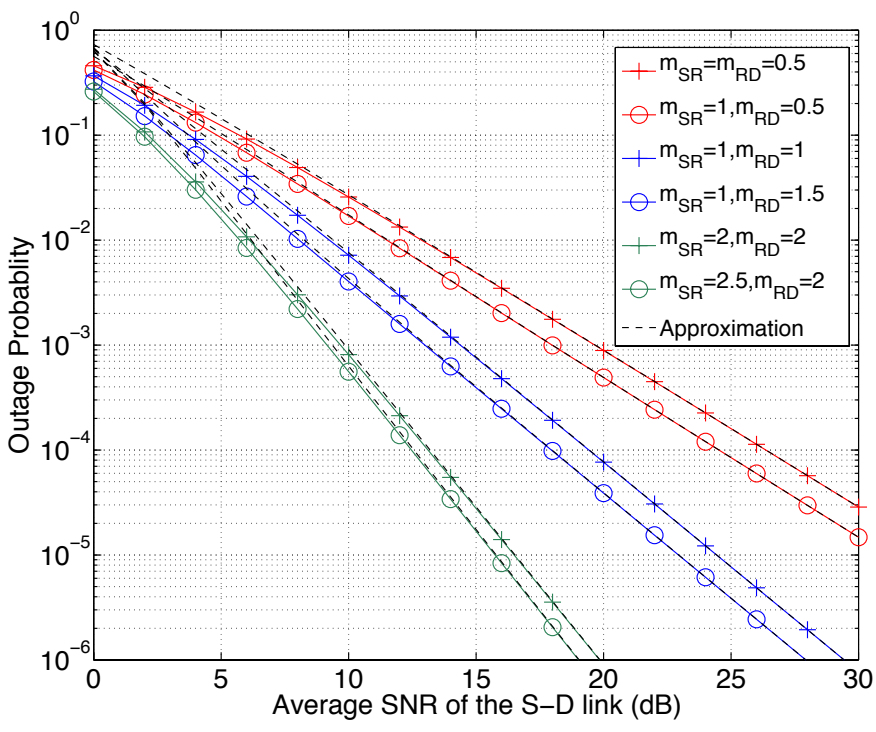

Fig. 7. Comparison of outage curves obtained by using the numerical calculation via (18), (19), and (20) and approximation via (21), (22), and (23), where $m_{\mathrm{SD}}=1$ and $G_{\mathrm{SD}}=G_{\mathrm{RD}}=G_{\mathrm{SR}}$.

(20) using a numerical integral method. From this observation, it can be concluded that the approximation is sufficiently accurate to calculate the outage probability.

Since $\bar{\gamma}_{\mathrm{SD}}, \bar{\gamma}_{\mathrm{RD}}$, and $\bar{\gamma}_{\mathrm{SR}}$ in (21), (22), and (23), are linear to a certain representative value $\bar{\gamma}$, and the exponent terms stay unchanged even if we replace $\bar{\gamma}_{\mathrm{SD}}, \bar{\gamma}_{\mathrm{RD}}$, and $\bar{\gamma}_{\mathrm{SR}}$ by $\bar{\gamma}$, we have

$$
\begin{aligned}
& P_{\mathrm{A}} \approx A \cdot A^{\prime} \cdot \bar{\gamma}^{\left(-m_{\mathrm{SD}}-m_{\mathrm{RD}}\right)}, \\
& P_{\mathrm{B}} \approx B \cdot \bar{\gamma}^{\left(-m_{\mathrm{SD}}-m_{\mathrm{SR}}\right)}, \\
& P_{\mathrm{C}} \approx C \cdot \bar{\gamma}^{\left(-m_{\mathrm{SD}}-m_{\mathrm{RD}}-m_{\mathrm{SR}}\right)} .
\end{aligned}
$$

It is observed from (27), (28), and (29) that, $\bar{\gamma}^{\left(-m_{\mathrm{SD}}-m_{\mathrm{RD}}-m_{\mathrm{SR}}\right)}$ is upper bounded by $\bar{\gamma}^{\left(-m_{\mathrm{SD}}-m_{\mathrm{RD}}\right)}$ or $\bar{\gamma}^{\left(-m_{\mathrm{SD}}-m_{\mathrm{SR}}\right)}$ when $\bar{\gamma}$ approaches infinity. Moreover, it is not difficult to find that $A^{\prime}$ equals one asymptotically at high SNR regime. Therefore, the overall outage probability of the LF relaying with Nakagami- $m$ fading can be formulated as

$$
P_{\text {out }}^{\mathrm{LF}}=\left(G_{c} \cdot \bar{\gamma}\right)^{\left(-G_{d}\right)} \text {, }
$$

where

$$
G_{d}=m_{\mathrm{SD}}+\min \left(m_{\mathrm{SR}}, m_{\mathrm{RD}}\right)
$$

and

$$
G_{c}= \begin{cases}\frac{1}{\sqrt[\left(G_{d}\right)]{B}}, & m_{\mathrm{SR}}<m_{\mathrm{RD}} \\ \frac{1}{\left(G_{d}\right)}, & m_{\mathrm{SR}}=m_{\mathrm{RD}} \\ \frac{1}{\left(G_{d}\right)}, & m_{\mathrm{SR}}>m_{\mathrm{RD}}\end{cases}
$$

are the equivalent diversity order $^{3}$ and coding gain [31] achieved by the LF relaying, respectively. We can see

${ }^{3} G_{d}$ indicates the decay of the outage probability curve versus average SNR $G_{i j} \frac{E_{s}}{N_{0}}$ because $G_{d}$ appears in the exponent part in (30). Therefore, the decay of the outage curve is concerned and it has the equivalent meaning as the diversity order. However, according to the definition, $G_{d}$ can take arbitrary real number which is related to the shape factor $m_{i j}$. In this sense, this paper calls the decay of the curve equivalent diversity order, which does not follow the standard definition of the diversity order. 


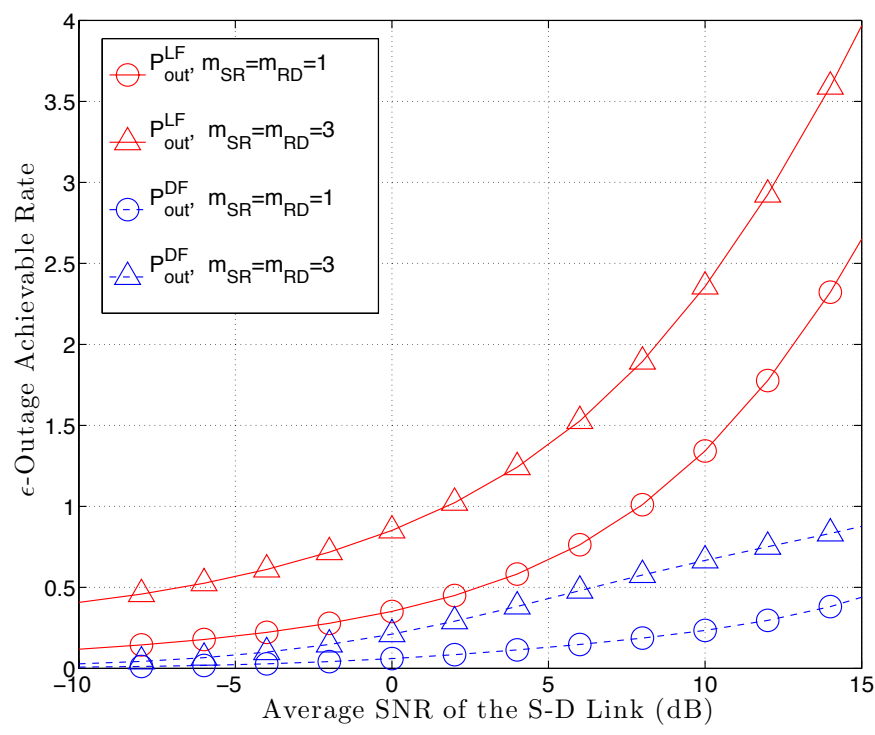

Fig. 8. The $\epsilon$-outage achievable rates of $\mathrm{LF}$ and $\mathrm{DF}$ for $\epsilon=0.01$, where $m_{\mathrm{SD}}=1$ and $G_{\mathrm{SD}}=G_{\mathrm{RD}}=G_{\mathrm{SR}}$.

from (31) that, the equivalent diversity order of the LF relaying system is restricted by the less reliable channel of either the S-R or R-D link. The outage curves shown in Fig. 7 also illustrate that the decay in outage curve cannot be obtained by only increasing the $m$ value of either the S-R or R-D link. The outage curves become sharper only when $m$ values of both the S-R and R-D links increase simultaneously, which is consistent to the conclusion drawn in (31) regarding equivalent diversity order.

\section{V. $\epsilon$-Outage Achievable Rate}

The $\epsilon$-outage achievable rate $R^{\epsilon}$ is defined as the largest achievable transmission rate $R_{c}$ such that the outage probability $P_{\text {out }}$ is not larger than a threshold $\epsilon$,

$$
R^{\epsilon}=\sup \left\{R_{c}: P_{\text {out }} \leq \epsilon\right\}, 0 \leq \epsilon \leq 1 .
$$

For LF and DF, the $\epsilon$-outage achievable rates $R_{\mathrm{LF}}^{\epsilon}$ and $R_{\mathrm{DF}}^{\epsilon}$ are, respectively, given by

$$
\begin{aligned}
& R_{\mathrm{LF}}^{\epsilon}=\sup \left\{R_{c}: P_{\mathrm{out}}^{\mathrm{LF}} \leq \epsilon\right\}, 0 \leq \epsilon \leq 1, \\
& R_{\mathrm{DF}}^{\epsilon}=\sup \left\{R_{c}: P_{\mathrm{out}}^{\mathrm{DF}} \leq \epsilon\right\}, 0 \leq \epsilon \leq 1,
\end{aligned}
$$

The $\epsilon$-outage achievable rates of the LF and DF relaying are illustrated in Fig. 8, where $\epsilon$ is set at 0.01. It is found that for both LF and DF, $R_{c}^{\epsilon}$ increases as the values of $m_{\mathrm{SR}}$ and $m_{\mathrm{RD}}$ increase. However, LF always outperforms DF in terms of $R_{c}^{\epsilon}$. It can also be observed from Fig. 8 that at high SNR region, the LF relaying is more advantageous than DF in terms of $\epsilon$-outage achievable rate.

\section{Optimal Relay Location}

Let $d_{\mathrm{SD}}, d_{\mathrm{RD}}$, and $d_{\mathrm{SR}}$ denote the distances between $\mathrm{S}$ and $\mathrm{D}, \mathrm{R}$ and $\mathrm{D}$, and $\mathrm{S}$ and $\mathrm{R}$ respectively. With $G_{\mathrm{SD}}$ being normalized to unity, $G_{\mathrm{SR}}$ and $G_{\mathrm{RD}}$ can be defined as $G_{\mathrm{SR}}=\left(\frac{d_{\mathrm{SD}}}{d_{\mathrm{SR}}}\right)^{\alpha}$ and $G_{\mathrm{RD}}=\left(\frac{d_{\mathrm{SD}}}{d_{\mathrm{RD}}}\right)^{\alpha}$, respectively, where $\alpha$

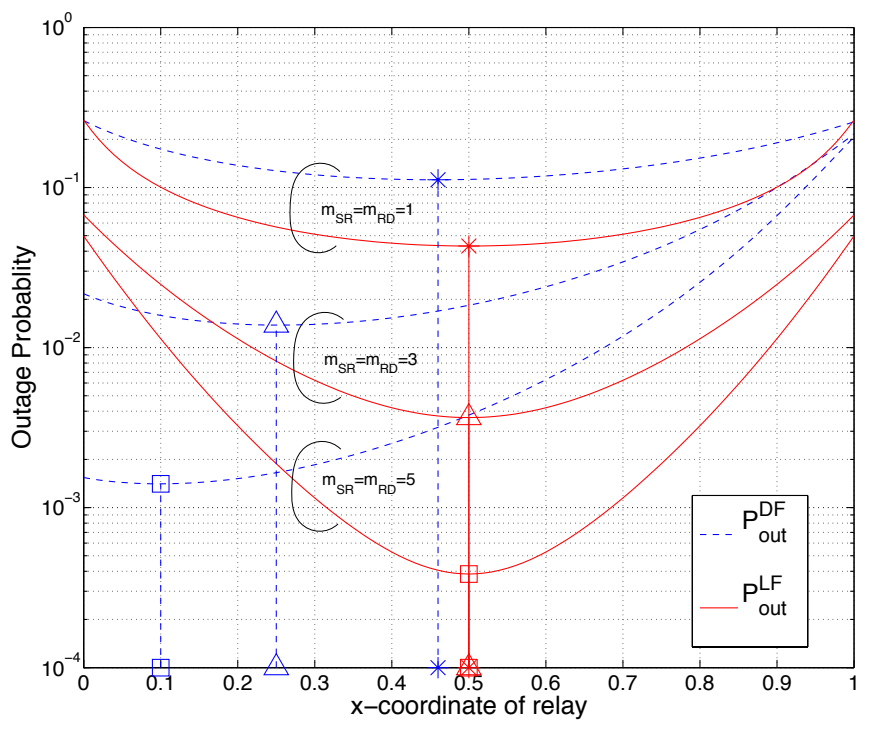

Fig. 9. The optimal relay positions of the $\mathrm{LF}$ and DF relaying where $m_{\mathrm{SD}}=$ 1 and $\alpha=3.52$. The vertical lines indicate the points where the outage probabilities are minimized.

is the path loss exponent. Then, the average SNRs of the S-R and R-D links can be given as

$$
\begin{gathered}
\bar{\gamma}_{\mathrm{SR}}=\bar{\gamma}_{\mathrm{SD}}+10 \log _{10}\left(G_{\mathrm{SR}}\right)(\mathrm{dB}), \\
\bar{\gamma}_{\mathrm{RD}}=\bar{\gamma}_{\mathrm{SD}}+10 \log _{10}\left(G_{\mathrm{RD}}\right)(\mathrm{dB}) .
\end{gathered}
$$

By substituting (36) and (37) into (18), (19), and (20), we can obtain the outage probability expression with respect to the position of R.

Fig. 9 shows the impact of the relay location on the outage probability, with $\bar{\gamma}_{\mathrm{SD}}=3 \mathrm{~dB}$. R is located on the line parallel to the S-D link between $x=0$ and $x=1$, as shown in Fig. 1 . The outage probabilities of the DF relaying, are also depicted as the references. With DF, the lowest outage probability can be achieved when $\mathrm{R}$ is located close to $\mathrm{S}$, while, interestingly, the lowest outage probability can be achieved when $\mathrm{R}$ is located at the midpoint with $\mathrm{LF}$ as long as $m_{\mathrm{SR}}=m_{\mathrm{RD}}$. It is also observed that the outage curves are symmetric with respect to the midpoint of the S-D link with the LF relaying. This is because with the LF relaying, the errors due to the S-R link can be corrected at the destination, and therefore, the midpoint $\left(d_{\mathrm{SR}}=d_{\mathrm{RD}}\right)$ is the optimal point where the contributions of the S-R and R-D links are balanced. Note that, for ensuring certain outage probability, the LF relaying has a larger range to find a relay than the DF relaying. 


\section{CONCLUSION}

The outage probability of the LF relaying has been analyzed over the independent Nakagami- $m$ fading channels. The exact expression of the outage probability has been derived. It has been found that through a reasonable approximation of the outage expression, the equivalent diversity order and coding gain of the LF relaying can be derived. It has been revealed that the equivalent diversity order (outage curve decay) is dominated by the less reliable channel of either the S-R or R-D link. Compared with the DF relaying, LF can achieve lower outage probability. Moreover, it has been found that the outage probability of the LF relaying derived from GCC and that from CCC are almost the same. The $\epsilon$-outage achievable rate of the LF relaying is always larger, compared with that of the DF relaying. Finally, it has been shown that since the LF relaying utilizes the contributions from both the S-R and $\mathrm{R}-\mathrm{D}$ links, the optimal $\mathrm{R}$ position is exactly in the midline between $\mathrm{S}$ and $\mathrm{D}$, so long as $m_{\mathrm{SR}}=m_{\mathrm{RD}}$. The observation about the relay location suggests that the search area for a relay (helper) can be increased by the LF relaying compared to the DF relaying.

Since the channel model practically depends on the position of nodes, joint analysis of the actual propagation property, the impact of node position, i.e., average SNR, and dynamic channel variation characteristics in terms of the higher order statistics is left for future study.

\section{REFERENCES}

[1] J. Laneman, D. Tse, and G. W. Wornell, "Cooperative diversity in wireless networks: Efficient protocols and outage behavior," IEEE Trans. Inform. Theory, vol. 50, no. 12, pp. 3062-3080, Dec 2004.

[2] P. Liu, Z. Tao, Z. Lin, E. Erkip, and S. Panwar, "Cooperative wireless communications: a cross-layer approach," IEEE Wireless Commun. Mag., vol. 13, no. 4, pp. 84-92, Aug 2006.

[3] S. Karmakar and M. K. Varanasi, "The diversity-multiplexing tradeoff of the dynamic decode-and-forward protocol on a MIMO half-duplex relay channel," IEEE Trans. Inform. Theory, vol. 57, no. 10, pp. 6569-6590, Oct 2011.

[4] M. Torbatian and M. O. Damen, "Diversity-multiplexing tradeoff of asynchronous decode-and-forward cooperative networks," IEEE Trans. Commun., vol. 62, no. 7, pp. 2340-2352, July 2014.

[5] Z. Cheng, N. Devroye, and T. Liu, "The degrees of freedom of fullduplex bidirectional interference networks with and without a MIMO relay," IEEE Trans. Wireless Commun., vol. 15, no. 4, pp. 2912-2924, April 2016.

[6] B. Zhao and M. C. Valenti, "Distributed turbo coded diversity for relay channel," Electron. Lett., vol. 39, no. 10, pp. 786-787, May 2003.

[7] P. Razaghi and W. Yu, "Bilayer low-density parity-check codes for decode-and-forward in relay channels," IEEE Trans. Inform. Theory, vol. 53, no. 10, pp. 3723-3739, Oct 2007.

[8] A. Stefanov and E. Erkip, "Cooperative coding for wireless networks," IEEE Trans. Commun., vol. 52, no. 9, pp. 1470-1476, Sept 2004.

[9] G. V. V. Sharma, V. Ganwani, U. B. Desai, and S. N. Merchant, "Performance analysis of maximum likelihood detection for decode and forward MIMO relay channels in rayleigh fading," IEEE Trans. Wireless Commun., vol. 9, no. 9, pp. 2880-2889, September 2010.

[10] G. Caire, G. Taricco, and E. Biglieri, "Optimum power control over fading channels," IEEE Trans. Inform. Theory, vol. 45, no. 5, pp. 1468 1489, Jul 1999

[11] J. Luo, R. S. Blum, L. J. Cimini, L. J. Greenstein, and A. M. Haimovich, "Link-failure probabilities for practical cooperative relay networks," in Proc. IEEE Veh. Technol. Conf., vol. 3, May 2005, pp. 1489-1493 Vol. 3.

[12] N. C. Beaulieu and J. Hu, "A closed-form expression for the outage probability of decode-and-forward relaying in dissimilar Rayleigh fading channels," IEEE Commun. Lett., vol. 10, no. 12, pp. 813-815, December 2006.
[13] Y. Wang, Y. Xu, N. Li, W. Xie, K. Xu, and X. Xia, "Relay selection of full-duplex decode-and-forward relaying over Nakagami-m fading channels," Electron. Lett., vol. 10, no. 2, pp. 170-179, 2016.

[14] K. Anwar and T. Matsumoto, "Accumulator-assisted distributed turbo codes for relay systems exploiting source-relay correlation," IEEE Commun. Lett., vol. 16, no. 7, pp. 1114-1117, July 2012.

[15] J. Garcia-Frias and Y. Zhao, "Near-Shannon/Slepian-Wolf performance for unknown correlated sources over AWGN channels," IEEE Trans. Commun., vol. 53, no. 4, pp. 555-559, April 2005.

[16] A. J. Aljohani, S. X. Ng, and L. Hanzo, "Distributed source coding and its applications in relaying-based transmission," IEEE Access, vol. 4, pp. 1940-1970, 2016.

[17] M. Cheng, K. Anwar, and T. Matsumoto, "Outage probability of a relay strategy allowing intra-link errors utilizing Slepian-Wolf theorem," EURASIP J. Advances Signal Processing, vol. 2013, no. 1, p. 34, 2013.

[18] T. M. Cover and J. A. Thomas, Elements of Information Theory, 2nd ed. USA: John Wiley \& Sons, Inc., 2006.

[19] A. E. Gamal and Y.-H. Kim, Network Information Theory. New York: Cambridge University, 2011.

[20] X. Zhou, M. Cheng, X. He, and T. Matsumoto, "Exact and approximated outage probability analyses for decode-and-forward relaying system allowing intra-link errors," IEEE Trans. Wireless Commun., vol. 13, no. 12, pp. 7062-7071, Dec 2014.

[21] A. Wolf, D. C. Gonzlez, J. C. S. S. Filho, and G. Fettweis, "Asymptotically optimal power allocation for wsns with mutually correlated sensing data," Electron. Lett., vol. 20, no. 7, pp. 1317-1320, 2016.

[22] S. Qian, M. Cheng, K. Anwar, and T. Matsumoto, "Outage probability analysis for correlated sources transmission over Rician fading channels," in Proc. IEEE Int. Symp. Pers., Indoor, Mobile Radio Commun., Sept 2013, pp. 1087-1091.

[23] M. Brulatout, H. Khalif, V. Conan, S. Szott, M. Natkaniec, K. KosekSzott, and L. Prasnal, "A cooperative MAC protocol for lossy forwarding networks," in Proc. Wireless Days Conf., March 2016, pp. 1-3.

[24] M. O. Hasna and M. S. Alouini, "Harmonic mean and end-to-end performance of transmission systems with relays," IEEE Trans. Commun., vol. 52, no. 1, pp. 130-135, Jan 2004.

[25] L. H. Ozarow, S. Shamai, and A. D. Wyner, "Information theoretic considerations for cellular mobile radio," IEEE Trans. Veh. Technol., vol. 43, no. 2, pp. 359-378, May 1994.

[26] D. Tse and P. Viswanath, Fundamentals of Wireless Communication. New York, NY, USA: Cambridge University Press, 2005.

[27] C. E. Shannon, "A mathematical theory of communication," Bell Syst. Tech. J., vol. 27, pp. 397-423, Jul. 1948.

[28] — - "Coding theorems for a discrete source with a fidelity criterion," Institute of Radio Engineers, International Convention Record, vol. 7, pp. 325-350, 1959.

[29] G.-C. Zhu and F. Alajaji, "Joint source-channel turbo coding for binary Markov sources," IEEE Trans. Wireless Commun., vol. 5, no. 5, pp. 1065-1075, May 2006.

[30] I. S. Gradshteyn and I. M. Ryzhik, Table of Integrals, Series, and Products, 7th ed. Burlington, MA, USA: Elsevier/Academic Press, 2007.

[31] Z. Wang and G. B. Giannakis, "A simple and general parameterization quantifying performance in fading channels," IEEE Trans. Commun., vol. 51, no. 8, pp. 1389-1398, Aug 2003. 